ARTIGO

Recebido em: $11 / 09 / 2018$

Aceito em:

$22 / 11 / 2018$

\title{
0 mapeamento dos repositórios institucionais brasileiros: perfil e desafios
}

\author{
The mapping of Brazilian institutional repositories: profile and \\ challenges
}

Simone da Rocha WEITZEL (sweitzel@unirio.br)*

*Professora da Universidade Federal do Estado do Rio de Janeiro do Departamento de Biblioteconomia e do Programa de Pós-graduação em Biblioteconomia - PPGB.

\begin{abstract}
Resumo
0 estudo tem por objetivo mapear os repositórios institucionais brasileiros até o período de maio de 2017 a fim de retratar a situação atual e contribuir com subsídios para orientar as ações e diretrizes nacionais e internacionais para implementação de repositórios ou sua integração em rede. 0 Estudo foi baseado em um levantamento exaustivo de repositórios brasileiros por meio de fontes específicas e pela observação direta. As análises foram baseadas nas variáveis: a) número de artigos e volume total de itens para identificar os repositórios alinhados ao Acesso Aberto Verde; b) tipos de financiamento recebidos além do apoio da própria instituição como indicadores de sua manutenção e permanência; c) verificação da qualidade dos repositórios por meio do indicador gerado pela fonte The Ranking Web of World Repositories que avalia visibilidade e impacto. Foi verificado que cerca de 54,5\% dos repositórios concentram 97,5\% do total de artigos dentre os 101 repositórios identificados no país. Um terço dos repositórios receberam financiamento direto ou indireto do governo brasileiro e representam $20 \%$ do total de artigos depositados. 0 total de 45,5\% dos repositórios foram qualificados no The Ranking Web of World Repositories. Apesar dos dados apontarem para a concentração de repositórios com artigos científicos cumprindo o estabelecido para a estratégia do Acesso Aberto Verde foram observadas dificuldades em relação ao apoio institucional e qualidade (visibilidade e impacto). 0 estudo recomenda que o governo brasileiro estabeleça diretrizes nacionais e políticas específicas para fomentar as boas práticas em repositórios no país com o objetivo de fortalecer o desenvolvimento do Acesso Aberto Verde.
\end{abstract}

Palavras-chave: Acesso Aberto Verde. Repositórios Instituicionais.

\begin{abstract}
This study aims to map Brazilian institutional repositories to build a profile of Green Open Access in the country until the period of May 2017 in order to portray the current situation and contribute with subsidies to guide national and international actions for the implementation of repositories or their integration in a network. This study is based on a comprehensive survey of Brazilian repositories through specific sources and direct observation. Analysis was based on the following variables: a) the number of articles and total volume of items to identify the repositories aligned with Green Open Access; b) whether the repository received funding from sources outside the institution as an indicator of its capacity for maintenance and resilience; and c) verification of the repository's quality, as indicated by its inclusion in The Ranking Web of World Repositories. It was found that $97.50 \%$ of the total articles are concentrated in only $54.50 \%$ of the 101 repositories identified in the country. One third of the repositories receive direct or indirect funding from the Brazilian government and represent $20 \%$ of the total of articles deposited. A total of $45.5 \%$ of the repositories were listed in The Ranking Web of World Repositories.Although the data indicate a concentration of repositories complying with Green Open Access, difficulties were observed in relation to institutional support and quality (visibility and impact). The study recommends that the Brazilian government establish national guidelines and specific policies to promote good practices in repositories in the country with the objective of strengthening the development of Green Open Access.
\end{abstract}

Keywords: Green Open Acess. Institutional Repositories.

v. 24, n. $54,2019$.

p. $105-123$

ISSN 1518-2924

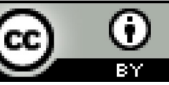

Esta obra está licenciada sob uma Licença Creative Commons. 


\section{INTRODUÇÃo}

A grande campanha mundial pelo Acesso Aberto à produção científica enunciada pela Budapest Open Access Initiative (2002) completou apenas dezesseis anos, mas já é possível verificar e desfrutar de seus benefícios em escala global. Mesmo sem ter cumprido as suas promessas e potencialidades com os patamares sonhados de $100 \%$ de acesso livre à produção científica sem qualquer barreira legal, técnica ou financeira, hoje, em qualquer parte do mundo, qualquer pessoa pode acessar por meio da internet à uma parcela dessa produção em texto completo estimado por Harnad em cerca de $25 \%$.

Além das mudanças e inovações no sistema de comunicação e produção científica a infra-estrutura estabelecida pelo Acesso Aberto viabilizou também as novas práticas científicas mais colaborativas tanto em relação à pesquisa quanto à produção científica, propriamente dita, fortalecendo as bases do surgimento do movimento pela Ciência Aberta um termo guarda-chuva "que engloba diferentes tipos de práticas e abordagens" científicas estabelecidas em rede, transparentes e responsáveis baseadas fortemente na cultura digital e no pressuposto de que o conhecimento científico é um bem público (ALBAGLI, 2015, p. 2 ; PORTUGAL, c2016).

A instituicionalização do Acesso Aberto no mundo se deu basicamente por meio de duas estratégias estabelecidas pela Declaração de Budapeste (BUDAPEST OPEN ACESS INITICATIVE, 2002) assim denominadas nos últimos anos: a) Acesso Aberto Dourado: baseado nos esforços da comunidade científica para produzir uma nova geração sustentada de periódicos eletrônicos sem cobrança de assinaturas ou taxas; b) Acesso Aberto Verde baseado no depósito do texto do artigo submetido a um periódico em um repositório institucional pelo próprio autor - seja a primeira versão seja a versão final já com a revisão pelos pares - conforme as orientações da política editorial da revista a que o autor submeteu o artigo.

Apesar dos revezes que afetaram o avanço do Acesso Aberto, especialmente em relação à monetarização do Acesso Aberto Dourado pelos grandes oligopólios representados pelas editoras comerciais que publicam periódicos de grande fator de impacto e cobram taxas de processamento de artigos (APCs - article processing charges), iniciativas de vários setores da comunidade científica - desde pesquisadores a instituições de ensino superior e pesquisa - somam esforços para a manutenção dos ideais estabelecidos na Declaração de Budapeste (BUDAPEST OPEN ACESS INITICATIVE, 2002), principalmente. O surgimento do COAR é um grande exemplo da vitalidade do Acesso Aberto Verde e suas ações visam alcançar uma nova geração de repositórios. Redes de repositórios vem sendo fortalecidas tais como RCAAP em Portugal, La Referencia na América Latina dentre outras iniciativas locais e nacionais que visam agregar esforços para alcançar a tão sonhada meta de $100 \%$ de acesso aberto.

Algumas fontes surgiram logo no início do chamado Movimento do Acesso Aberto especialmente para monitorar o desenvolvimento dos repositórios ao redor do mundo tal como por exemplo a fonte Directory of Open Access Repositories (OpenDOAR) que contém em sua base mais de 3.300 repositórios segundo dados de maio de 2017 - um dos maiores diretórios de repositórios do mundo.

No entanto, essas fontes são dependentes do cadastramento voluntário pelo gestor do repositório. Cada diretório contém suas regras, requisitos e critérios próprios para que o repositório seja incluído e permitindo que seus dados atualizados frequentemente de forma automática, especialmente em relação ao conteúdo dos repositórios cuja tendência é aumentar em termos de itens colecionados. Assim, em função da necessidade de cadastramento para fazer parte do monitoramento automático, é possível que uma parcela de repositórios existentes não esteja listada nos Diretórios. Outros problemas agravam a situação. Em consultas simples nesses diretórios percebe-se que alguns repositórios listados não existem mais ou seus dados não estão atualizados talvez por problemas técnicos relativos ao protocolo de intercâmbio de metadados, entre outros problemas. Há também dados duplicados (dupla entrada), links quebrados, entre outros problemas afetando a missão desses diretórios em retratar de forma mais exaustiva possível o panorama dos repositórios no mundo.

Considerando esses fatores o presente estudo tem por objetivo mapear os repositórios institucionais brasileiros até o período de maio de 2017 a fim de retratar a situação atual em relação ao seu potencial de cumprir ao que foi estabelecido pela estratégia 
do Acesso Aberto Verde na BOAI e contribuir com subsídios para orientar a gestão de repositórios bem como ações e diretrizes nacionais e internacionais para implementação e desenvolvimento de repositórios ou sua integração em rede.

A relevância deste estudo se destaca pela necessidade de valorizar e estimular o Acesso Aberto Verde no país promovendo o desenvolvimento da ciência e de estimular outros estudos que possam colaborar para o fomento de políticas nacionais para repositórios inclusive de ações mais efetivas para mapear os repositórios brasileiros de forma exaustiva e sistemática.

0 estudo baseou-se em um amplo levantamento em fontes específicas, a saber: OpenDOAR, ROARMAP, ROAR, Lista de repositórios do IBICT, resultado do edital da FINEP que apoiou a implantação de repositórios no país, lista de usuários do DSPACE bem como repositórios listados no The Ranking Web of World Repositories (2017).

Cada repositório foi consultado por meio da observação direta para verificar se o repositório contem artigos de periódicos - o indicador adotado neste estudo para traduzir o alinhamento do repositório à estratégia do Acesso Aberto Verde estabelecido no BOAI.

Dentre os principais resultados deste estudo foi verificado que cerca de 56 repositórios - representando 54\% do total identificado - concentra $97 \%$ do total de artigos de todos os repositórios em funcionamento do país. Dos 33 repositórios que receberam o financiamento da FINEP, 20 repositórios concentram praticamente toda a produção de artigos daqueles repositórios embora represente apenas 20\% do total de artigos dos 101 repositórios identificados.

0 estudo também evidenciou diversas lacunas de ordem gerencial nos repositórios brasileiros que prejudicam a geração de indicadores nacionais e internacionais reivindicando políticas específicas para fomentar as boas práticas e estabelecer diretrizes nacionais.

\section{ACESSO ABERTO VERDE: ESTRATÉGIAS E DESAFIOS}

O debate sobre Acesso Aberto no Brasil é certamente complexo e pode ser visto sob várias dimensões. Neste artigo prevaleceu a contextualização do novo cenário para a produção científica de países emergentes como o Brasil, breve histórico do surgimento do acesso aberto no país com ênfase na abordagem do Acesso Aberto Verde bem como os desafios que a Região da América Latina vem enfrentando a partir dos estudos levantados. Também foram identificadas as principais categorias teóricas que envolvem ou afetam o Acesso Aberto os quais se articulam com as variáveis apresentadas na seção 3.

\subsection{Um novo cenário para a produção científica de países emergentes}

Guédon (2010, p. 22) avalia que a estrutura do poder científico na atualidade foi fortemente afetada pelo Acesso Aberto beneficiando particularmente os países pobres e em desenvolvimento que passaram a ter acesso aos resultados de pesquisa do mundo todo sem precisar pagar por isso. No entanto, o autor considera que o caminho a ser percorrido ainda é bastante longo - é preciso traçar estratégias para superar a estrutura que separa a ciência produzida por países ricos - representada pela ciência predominante ou central (mainstream) - da ciência denominada de periférica produzida pelos países pobres e em desenvolvimento.

Ainda nos dias de hoje pesquisadores não conseguem ter seus artigos publicados em periódicos de alto fator de impacto, seja pela concorrência internacional, seja pelos recursos financeiros necessários para publicar nessas revistas em função das taxas de processamento de artigos (APCs). Questões que envolvem o idioma local, investimento em pesquisa dentre outros elementos também afetam os melhores posicionamentos dos países pobres e em desenvolvimento nas atividades de pesquisa quando comparados aos países ricos.

Em função dessas dificuldades Guédon $(2010,2004)$ destaca o potencial dos repositórios institucionais para elevar a visibilidade e impacto dos resultados das pesquisas dos países que produzem o que ele denomina de ciência periférica sem onerar o custo de manutenção e, sobretudo, para promover a mais ampla forma de inteligência distribuída no planeta.

O Blog do Open Access Scholarly Information Source (2010) acrescenta outros benefícios: a) incorpora a pesquisa local em igualdade de condições a partir de uma rede 
interoperável de conhecimento global; b) promove o estabelecimento de parcerias superando o isolamento profissional; c) promove o fortalecimento de uma base científica nacional e independente otimizando os investimentos realizados na pesquisa.

São possibilidades viabilizadas pelas conquistas trazidas pelo movimento do Acesso Aberto que, de acordo com Tzoc (2012), também poderão contribuir para fomentar diversificação de idiomas em publicações cientificas.

Um exemplo real dessas possibilidades está no protagonismo dos países emergentes denominados de BRICS (Brasil, Russia, Índia, China e Africa do Sul) devido a sua crescente participação global em atividades de pesquisa e desenvolvimento. Além de representar juntos a metade da população do mundo e $22 \%$ da economia mundial, os países da BRICS foram responsáveis por $24 \%$ das publicações científicas indexadas na base Scopus. China se destaca dentre os países da BRICS com mais artigos que os países do Reino Unido, França e Alemanha juntos conforme verificou o estudo de Joachim Schöpfel (2017).

Apesar do mercado internacional de publicações científicas ser atualmente dominado pelos modelos de publicação de editores americanos, britânicos, holandeses e alemães, Schöpfel (2017) defende a hipótese de que no futuro esse domínio exercido atualmente por grandes corporações será compartilhado por esses países emergentes que estarão mais bem posicionados no mercado internacional inclusive para oferecer modelos de publicação sustentáveis para países da América Latina e África do Norte e Subsaariana.

\subsection{Estratégias para a introdução do Acesso Aberto Verde: o caso brasileiro}

0 caso brasileiro tem sido reconhecido internacionalmente por suas estratégias pioneiras e bem-sucedidas na introdução do Acesso Aberto no país influenciando até mesmo o desenvolvimento do Acesso Aberto no mundo conforme afirmam autores e lideranças do Movimento do Acesso Aberto mundial (ALPERIN; FISCHMAN; WILLINSKY, 2008; HARNAD; CARR; GINGRAS, 2008).

As ações do Centro Latino-Americano e do Caribe de Informação em Ciências da Saúde (Bireme) e do Instituto Brasileiro de Informação em Ciência e Tecnologia (IBICT) inauguraram as principais iniciativas que introduziram e fortaleceram o acesso aberto no Brasil. Suas estratégias diferenciadas contribuíram para que o país alcançasse grande projeção no Movimento de Acesso Aberto com conteúdo brasileiros e da Região da América Latina e Caribe principalmente.

É possível afirmar que o Programa Scielo e Rede Scielo da Bireme fortaleceram o Acesso Aberto Dourado com seu pioneirismo em promover o acesso às revistas científicas brasileiras com ações desde 1998 que desencadearam o aperfeiçoamento do modelo de publicação, de indicadores bibliométricos e de coleções de periódicos de vários países iberoamericanos e da Africa do Sul e, mais recentemente, da Rede Scielo Livros (PACKER, MENENGUINI, 2014).

Já o Acesso Aberto Verde teve o IBICT como principal articulador de seu desenvolvimento sendo este último, por essa razão, foco desta revisão.

O IBICT promoveu diversas ações com o objetivo de implementar a infraestrutura necessária para apoiar e promover o acesso aberto em larga escala e de forma integrada em conformidade com os modelos existentes e amplamente adotados no mundo todo. As lideranças das universidades e institutos de pesquisa foram envolvidas no processo a fim de garantir o acesso às tecnologias e recursos requeridos tanto para publicação digital quanto para a implementação de repositórios institucionais conforme relatos de Costa, Kuramoto e Leite (2013, p. 134). Os autores consideram que as ações relativas ao Acesso Aberto no Brasil iniciam a partir de 2003 quando seus representantes participaram da 7. ELPUB - The International Conference on Electronic Publishing - ocorrido em 2003 em Portugal.

As iniciativas inovadoras das plataformas digitais para publicação de periódicos e de implantação de repositórios apresentadas naquele evento influenciaram enormemente as estratégias desenvolvidas nos anos seguintes pelo IBICT orientando as "ações relacionadas ao movimento global de acesso aberto à informação científica" no país (COSTA; KURAMOTO; LEITE, 2013, p. 134).

Dentre as ações implementadas destacam-se a tradução e treinamento das principais plataformas digitais para publicação de periódicos e anais de congressos bem como da implementação de repositórios - respectivamente o Open Journal System (traduzido para o português como Sistema Eletrônico de Editoração de Revistas - SEER com mais de 1.500 títulos de periódicos); Open Conference System (traduzido para o português 
como Sistema Online de Acompanhamento de Conferências - SOAC); e DsPACE - software adotado para implementação de repositórios.

Diretórios e provedores de serviços foram constituídos pelo IBICT ao longo dos últimos quinze anos a fim de fortalecer a infraestrutura requerida para integração dos provedores de dados dentre os quais destacam-se:

a) Portal Brasileiro de Acesso Aberto à Informação Científica (OASIS.BR http://oasisbr.ibict.br) - reúne títulos de revistas científicas e repositórios brasileiros;

b) Diretório de Políticas de Acesso Aberto das Revistas Científicas Brasileiras (DIADORIM - http://diadorim.ibict.br) - é uma iniciativa similar ao Projeto SHERPA/ROMEO;

c) Diretório Luso-Brasileiro (http://diretorio.ibict.br) - reúne as fontes de informação científica de acesso aberto produzidas por Brasil e Portugal;

d) Portal do Livro Aberto em C\&T (http://livroaberto.ibict.br) - reúne as publicações oficiais em ciência, tecnologia e inovação do país.

e) La Referencia: Rede Federada de Repositórios Institucionais de Publicações Científicas. Surge em 2012 a partir de um Acordo de Cooperação assinado em Buenos Aires entre os países da Argentina, Brasil, Chile, Colômbia, Costa Rica, Equador, El Salvador, México e Peru para formar uma rede latino-americana de Acesso Aberto. Possui mais de um milhão de textos completos de artigos, relatórios e teses e dissertações (LA REFERENCIA, [2018]).

Dentre os eventos promovidos pelo IBICT sobre o tema destaca-se a Conferência Luso-Brasileira de Acesso Aberto (CONFOA). 0 evento, realizado anualmente de forma alternada no Brasil e em Portugal, reúne pesquisadores brasileiros e portugueses em torno das questões sobre Acesso Aberto e Ciência Aberta. A comissão organizadora da ConfOA é formada pela Fundação para a Ciência e a Tecnologia - FCT (Portugal), Universidade do Minho (Portugal) e o IBICT (Brasil) conforme "ações decorrentes do Memorando de Entendimento assinado entre os Ministros da Ciência e Tecnologia de Portugal e do Brasil em outubro de 2009" (INSTITUTO BRASILEIRO DE INFORMAÇÃO EM CIÊNCIA E TECNOLOGIA, 2017).

O lançamento de dois manifestos em defesa das ações relativas à adoção do acesso aberto no país foram importantes para sensibilizar a ampla participação dos agentes públicos e privados em torno da questão: Manifesto Brasileiro de Apoio ao Acesso Livre à Informação Científica de 2005 (INSTITUTO BRASILEIRO DE INFORMAÇÃO EM CIÊNCIA E TECNOLOGIA, 2005) e o Manifesto de Acesso Aberto a Dados da Pesquisa Brasileira para Ciência Cidadã de 2016 (INSTITUTO BRASILEIRO DE INFORMAÇÃO EM CIÊNCIA E TECNOLOGIA, 2016).

Editais da Finep em convênio com o IBICT foram lançados em 2009 e também tiveram grande relevância para o financiamento e fortalecimento tanto o Acesso Aberto Dourado quanto Verde no país. As instituições contempladas pelo edital receberam "kits tecnológicos" e apoio técnico-operacional com o objetivo de superar as dificuldades em relação à capacitação de recursos humanos e de infra-estrutura que afetavam a implementação do sistema de publicação digital e de repositórios. Cada kit continha um servidor com os softwares necessários para a implementação de repositórios institucionais com a plataforma DSPACE instalada bem como outros softwares livres com recursos para apoiar a gestão, publicação e o armazenamento em meio digital de publicações científicas (teses e dissertações, revistas científicas e anais de eventos). Essa estratégia contemplou inicialmente 27 instituições de ensino superior e de pesquisa no primeiro edital totalizando 33 instituições com lançamento de um segundo edital. Posteriormente foram realizados dois eventos para auxiliar a implementação dessas tecnologias: a) I Encontro sobre Gestão de Repositórios Institucionais realizado em 2009 que teve por objetivo coordenar as atividades das instituições contempladas pelos editais; b) I Workshop para Construção de Repositórios Institucionais a fim de capacitar os profissionais e técnicos das instituições que receberam os "kits tecnológicos" realizado em Brasília em julho de 2010 (BLOG..., 2010; INSTITUTO BRASILEIRO DE INFORMAÇÃO EM CIÊNCIA E TECNOLOGIA, 2009, 2010). Esses esforços conjugados - de um lado as ações da Bireme envolvendo a Região da América Latina com o Programa Scielo e de outro as ações do IBICT focadas no país - resultaram na liderança do Brasil no Movimento do Acesso Aberto. 


\subsection{Acesso Aberto Verde: os desafios da Região da América Latina e do caso brasileiro}

Apesar dos avanços obtidos em torno do Acesso Aberto para Costa e Leite (2017) e Santillán Aldana (2014) o país - especialmente a Região da América Latina e Caribe - ainda não conseguiu alavancar o Acesso Aberto Verde nem tão pouco alcançou o mesmo êxito que o acesso aberto dourado. Características próprias da Região, tais como a predominância do financiamento público das pesquisas e a baixa presença de editores científicos comerciais, vem beneficiando mais o desenvolvimento do Acesso Aberto Dourado que o Verde segundo esses autores.

A diferença pode ser verificada com os dados provenientes do Acesso Aberto Dourado e Verde no Brasil. O país possui $10 \%$ de títulos de periódicos em acesso aberto dourado do total cadastrado no Directory of Open Access Journals (dados extraídos em abril de 2017) superando países como Reino Unido (9\%), Estados Unidos (6\%) e Espanha (5,5\%). A situação se inverte em relação ao total de repositórios cadastrados no OpenDOAR onde o país está representado com pouco menos de 3\%. Estados Unidos lideram o ranking com quase $15 \%$ seguido pelo Reino Unido (7,5\%) e Alemanha (com cerca de 6\%). A proporção e liderança não difere quando comparada aos números de repositórios registrados no ROAR: Estados Unidos com pouco mais de 17\%, Reino Unido com quase 6\% e Alemanha com 5\%. 0 Brasil aparece com 3,5\% e apesar dos números pouco expressivo ocupa atualmente os 8 lugares em número de repositórios no mundo conforme dados do OpenDOAR.

Já o caso do Acesso Aberto Verde a grande diferença em termos percentuais entre o Brasil e os Estados Unidos (entre 12 e 15\%) evidenciam a necessidade de investimentos para aumentar o número de repositórios em pleno funcionamento e, sobretudo do número de artigos presentes nesses repositórios. Mesmo com repositórios brasileiros recebendo a produção proveniente dos periódicos de acesso aberto - cerca de $62 \%$ de acordo com o estudo de três casos típicos de Brumatti (2016, p. 100) - é preciso considerar a importância de concentrar artigos de periódicos em repositórios para fazer cumprir a missão do Acesso Aberto Verde potencializando a visibilidade e o impacto.

As principais tendências do Acesso Aberto Verde na região podem ser observadas no estudo de Costa e Leite $(2017$, p. 131, 133, 161) que verificou 84 repositórios institucionais (RI) da Região da América Latina e Caribe (incluindo México) destacando as seguintes características: a) predominância de artigos científicos em RI (36\%); b) teses, dissertações e trabalhos de conclusão de curso (24\%) e documentos administrativos e arquivísticos (24\%) também são bastante expressivos (embora não sejam contemplados pelos preceitos do Acesso Aberto Verde); c) trabalhos apresentados em eventos são pouco representativos (9\%) assim como livros e capítulos de livros (2\%); d) predominância da área das Ciências Exatas e Naturais (três vezes maior que a área das Ciências Sociais e Humanas que, por sua vez, é quatorze vezes maior que a área de Artes e Humanidades); e) supremacia do DSPACE entre os países da Região; f) as políticas institucionais dos RIs representam quase $18 \%$ do total de RIs analisados no estudo - valor considerado insuficiente pelos autores; g) os países com mais conteúdo em RIs são Brasil (46\%), Colômbia (17\%), Argentina (13\%) e México (9\%) que juntos representam $85 \%$ dos conteúdos da América Latina; h) A média de documentos depositados em repositórios na Região é de 7 mil documentos.

É curioso observar que os estudos realizados para mapear o acesso aberto na Região da América Latina e Caribe demonstraram que os periódicos de acesso aberto estão concentrados nas áreas de Ciências Sociais Aplicadas (SANTILLÁN ALDANA, 2014) enquanto que os repositórios reúnem majoritariamente a produção científica das Ciências Exatas e Naturais (COSTA; LEITE, 2017; BRUMATTI, 2016, p. 87).

Por outro lado, os dados do World Research 2015 indicam que o total da produção científica de oito países da Região (Argentina, Brasil, Chile, Colômbia, Cuba, México, Peru e Venezuela) estão concentrados nas Ciências Agrárias (ELSEVIER, 2015). Logo, é possível inferir que uma parte da produção científica estaria sendo publicada ou depositada fora dos países de origem e/ou da Região.

Babini e Machin-Mastromatteo (2015) observaram que pesquisadores independentes e pesquisadores lotados em instituições que não possuem repositórios realizam o depósito de sua produção científica publicada em periódicos comerciais em repositórios temáticos os quais em sua maioria são estrangeiros. 0 estudo de Brumatti (2016, p. 100) aponta para essa tendência ao verificar em uma amostra que $38 \%$ do total da produção científica oriunda de três programas de pós-graduação (física, economia e 
psicologia) de três universidades brasileiras entre 2013 e 2014 estava depositada em repositórios estrangeiros.

Para concentrar a produção no próprio país de origem Babini e MachinMastromatteo (2015) recomendam a implementação de repositórios nacionais embora reconheçam a complexidade dessa proposta. Seria preciso desenvolver um sistema para controle de qualidade dos documentos depositados bem como políticas adequadas e estrutura legal.

Dessa forma, para que o acesso aberto continue a ser desenvolvido na Região da América Latina e Caribe, Babini e Machin-Mastromatteo (2015) defendem o estabelecimento de políticas, a promoção de boas práticas, treinamento de interessados, investimento em infraestrutura e especialmente a valorização da produção científica publicada em periódicos de acesso aberto nacional e regional garantindo que mudanças necessárias ocorram.

Costa e Leite (2017) também destacaram a importância da formalização de políticas e programas nacionais de acesso aberto e fomento do debate público sobre o tema para fortalecer o Acesso Aberto Verde na Região.

Os dados apresentados nesses estudos demonstram a importância de se fazer levantamentos nacionais e regionais a fim de verificar a distribuição da produção científica em periódicos (de acesso aberto, os comerciais, e aqueles que exigem pagamento para publicar) e em repositórios sejam institucionais ou temáticos (nacionais ou estrangeiros) de forma que os resultados possam orientar as políticas para o Acesso Aberto em cada país e, se possível, na Região da América Latina.

A pesquisa descrita a seguir foi realizada em repositórios brasileiros e procurou contribuir para o retrato desse cenário.

\subsection{Categorias teóricas}

A partir da literatura consultada foram construídas três categorias teóricas que se correlacionam com as variáveis do estudo conforme está descrito na seção 3 , a saber: acesso aberto, problemas que afetam o acesso aberto e tipos de abordagens do acesso aberto.

\subsubsection{Acesso aberto}

Neste trabalho acesso aberto se refere ao que foi estabelecido pelo BOAI conforme segue:

"Acesso aberto" à literatura científica revisada por pares significa a disponibilidade livre na Internet, permitindo a qualquer usuário ler, fazer download, copiar, distribuir, imprimir, pesquisar ou referenciar o texto integral desses artigos, recolhe-los para indexação, introduzi-los como dados em software, ou usá-los para outro qualquer fim legal, sem barreiras financeiras, legais ou técnicas que não sejam inseparáveis ao próprio acesso a uma conexão à Internet. As únicas restrições de reprodução ou distribuição e o único papel para o direito autoral neste domínio é dar aos autores o controle sobre a integridade do seu trabalho e o direito de ser devidamente reconhecido e citado.

0 termo Acesso Aberto Verde foi adotado neste estudo para designar a estratégia estabelecida pela Declaração de Budapeste (BUDAPEST OPEN ACESS INITIATIVE, 2002) que envolve o depósito da produção científica em um repositório conforme designado por Harnad (2012) e Gargouri e outros ([2012?] em lugar do termo Via Verde anteriormente adotado por Harnad e outros (2004).

\subsubsection{Problemas que afetam o Acesso Aberto}

De acordo com Harnad dois grandes problemas afetam o Acesso Aberto Verde na atualidade (GIGLIA; HARNAD, 2012):

a) o baixo e lento crescimento do número de políticas mandatórias adotadas pelas instituições produtoras de conhecimento científico e/ou acadêmico ao redor do mundo. As políticas mandatórias contribuem para o fortalecimento dos repositórios com conteúdo de relevância na medida em que torna obrigatório o depósito da produção científica da comunidade universitária e/ou de pesquisadores de cada instituição de ensino superior ou de pesquisa que mantém seus respectivos repositórios institucionais. As recomendações para os próximos dez anos da BOAI mantém a estratégia da política mandatória especialmente das "versões revisadas por pares, de todos os futuros artigos científicos da 
autoria dos seus membros" a fim de assegurar o depósito imediato em repositórios institucionais.

b) a desvalorização do Acesso Aberto Verde por vários setores da indústria da informação global, especialmente os editores comerciais de periódicos, com a defesa da "monetarização" do Acesso Aberto Dourado por meio de cobranças de taxas para custear o processamento de artigos de periódicos científicos (APCs). Essas taxas são pagas pelo autor ou pela instituição de origem para permitir a publicação de artigos em acesso aberto e "sustentar" esse modelo. Harnad denomina esse modelo de Acesso Aberto Dourado híbrido pois os editores continuam a oferecer a assinatura convencional de seus títulos como antes e cobra uma taxa extra para aqueles que desejam publicar os artigos "em acesso aberto".

$\mathrm{O}$ acesso aberto dourado, conforme visto, é originalmente uma estratégia da BOAI e está baseada na produção de uma nova geração de periódicos que não cobra nem para publicar nem para acessar (anteriormente denominado de Via Dourada). No entanto, a indústria da informação global se apropriou do Acesso Aberto Dourado com fins lucrativos e desvaloriza o Acesso Aberto Verde como estratégia para o acesso aberto. No Reino Unido, por exemplo, o conhecido Relatório Finch - documento elaborado por uma comissão independente liderada por Dame Janet Finch e apresentada à Câmara Alta do Reino Unido deslocam o papel dos repositórios para figurante e complementar cuja função se resume a preservar e promover o acesso à dados científicos e à literatura cinzenta (FINCH et al., 2012) afetando as estratégias seminais do acesso aberto.

\subsubsection{Tipo de Abordagens}

Considerando que o objetivo deste artigo é mapear os repositórios institucionais brasileiros e verificar a situação atual em relação ao que foi estabelecido no BOAI especificamente quanto ao Acesso Aberto Verde, isto é, que os repositórios institucionais devem conter artigos de periódicos em seu conteúdo, é importante apresentar as duas abordagens denominada de rígida e flexível conforme define Leite (2009, p. 27-30). Na abordagem rígida os repositórios priorizam conteúdos revistos por pares especialmente os artigos de periódicos com a finalidade de maximizar os impactos dos resultados das pesquisas conforme preconizado pelo Movimento do Acesso Aberto. Já na abordagem flexível, os repositórios contemplam além dos conteúdos avaliados por pares, todos os demais de caráter acadêmico ou científico mesmo que não tenha sido avaliado por pares. Contudo, a apropriação pelas comunidades científica e acadêmica das estratégias e infraestrutura oferecida pelo Movimento do Acesso Aberto diminuiu essas fronteiras que identificavam essas abordagens trazendo para o cenário atual um sem número de possibilidades de coleções em repositórios que incluem desde textos acadêmicos, administrativos bem como obras com imagens em movimento ou paradas provenientes dos setores culturais, entre outros.

\section{MATERIAL E MÉTODOS}

Esta pesquisa, de caráter descritivo e exploratório, foi delineada a partir do estudo de caso dos repositórios brasileiros em três etapas para retratar o número mais real possível de repositórios em funcionamento no país até maio de 2017: 1ํำ identificação do Universo

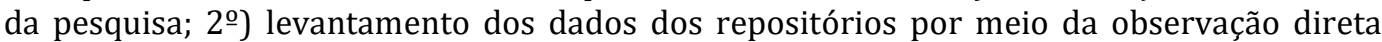
conforme 4 variáveis: número de artigos, volume total de itens, tipo de financiamento, e indicador de qualidade (visibilidade e impacto); $3^{\circ}$ ) Coleta, Tabulação, discussão e análise dos dados. As fontes que foram foco do estudo também estão brevemente descritas a seguir

\subsection{Etapas da pesquisa de campo}

$1^{\text {o }}$ ) identificação do Universo da pesquisa: foi realizado um amplo levantamento em fontes de informação na internet que listam e descrevem repositórios em todo o mundo. Como os dados diferem em termos quantitativos e qualitativos esta etapa da pesquisa foi importante para mapear de forma exaustiva o número de repositórios existentes efetivamente no país. Também é importante mencionar que os diretórios, especialmente, são constituídos por informações dadas por colaborações voluntárias dos gestores de repositórios e, por isso, é possível que o número de repositórios e de seus conteúdos seja maior. As fontes consultadas foram: OpenDOAR, ROARMAP, ROAR, Lista de repositórios do IBICT, documento contendo o resultado do edital da FINEP que apoiou a implantação de 
repositórios no país, lista de usuários do DSPACE bem como repositórios listados no The Ranking Web of World Repositories com a finalidade de mapear o universo da pesquisa.

$2^{\mathrm{o}}$ ) levantamento dos dados dos repositórios: cada repositório identificado na etapa anterior foi consultado por meio da observação direta, para levantar as seguintes variáveis:

a) número de artigos e volume total de itens: para verificar através da proporção de artigos se o repositório cumpre sua missão em relação ao Acesso Aberto Verde ou se, de fato, os repositórios armazenam preferencialmente outros tipos de materiais conforme sugere o Relatório Finch e outros (2012). Neste caso, o número de artigos de periódicos deve ter uma proporção significativa em relação aos demais tipos de coleções do repositório;

b) tipo de financiamento: para verificar se o repositório recebeu algum tipo de financiamento além do apoio da própria instituição. Essa variável está relacionada com a premissa de que o sucesso dos repositórios é definido pelos subsídios que recebem e tem por objetivo funcionar como um indicador de fortalecimento dos repositórios em termos de sua manutenção e permanência;

c) indicador de qualidade (visibilidade e impacto): verificar se o repositório foi ranqueado ou não pela fonte The Ranking Web of World Repositories na edição, disponível no momento da pesquisa (janeiro de 2017) atribuindo, de alguma forma, qualidade e relevância aos repositórios no cenário internacional por meio dos indicadores web que são usados para medir visibilidade global e impacto. A fonte é uma iniciativa do Cybermetrics Lab um grupo de pesquisa oriundo do Consejo Superior de Investigaciones Científicas (CSIC) da Espanha. Os repositórios presentes nessa fonte são considerados qualificados. Já os ausentes serão considerados não-qualificados.

3ํ) Coleta, Tabulação, discussão e análise dos dados para confrontar as variáveis realizar um balanço do estudo.

\subsection{Descrição das fontes utilizadas na pesquisa}

A pesquisa fez uso de cinco fontes a saber: OpenDOAR, ROAR, Lista de repositórios brasileiros do IBICT, Lista das universidades contempladas pelo Edital da Finep, Lista de repositórios cujas instituições são usuários do software DSPACE e ranking do Cybermetrics Lab conforme segue abaixo. As fontes foram importantes para identificação do universo de repositórios no país.

a) Diretórios de repositórios

O OpenDOAR e o ROAR são diretórios de repositórios que surgiram para apoiar análises quantitativas e estatísticas bem como o desenvolvimento de políticas e padrões para a comunidade envolvida com repositórios (CALHOUN, 2014, p. 92). Millington (2008) acrescenta outros propósitos envolvendo as boas práticas de gestão além de indicadores estatísticos bem como a possibilidade de busca em seus conteúdos e de fornecer listas para harvesters e data mining. O projeto do OpenDOAR surgiu em 2005 e foi lançado em 2006 em parceria com University of Nottingham no âmbito do projeto Sherpa e Lund University com apoio da OSI, JISC, SPARC Europe e CURL (MILLINGTON, 2008). Já o ROAR é um projeto da Rede Eprints.org da University of Southampton no Reino Unido que surgiu em 2003 de acordo Calhoun (2014, p. 90). A autora reconhece que nem todos os repositórios existentes no mundo estão registrados nessas fontes e, por isso, o número de repositórios e de itens colecionados ainda é desconhecido (CALHOUN, 2014, p. 92).

b) Listas

Três listas foram consultadas como fonte para identificação de repositórios brasileiros: "Lista de repositórios brasileiros" compilada pelo IBICT e disponível em seu site; 0 documento contendo o resultado do edital da FINEP que apoiou a implantação de repositórios no país listando as universidades contempladas; e a Lista de usuários do DSPACE obtidos na interface de busca por país (DSPACE FOUNDATION, [2010]).

c) Ranking do Cybermetrics Lab

0 ranking de repositórios The Ranking Web of World Repositories foi desenvolvido pela Cybemetrics Lab do Consejo Superior de Investigaciones Científicas (2012) na Espanha. De acordo com Carvalho (2018) esteve ativo no período entre 2008 e 2017 quando foi desativado por seus idealizadores. 


\section{RESULTADOS E DISCUSSÃO}

A partir da consulta e verificação dos links dos repositórios listados em cada fonte consultada foram identificados o total de 130 repositórios brasileiros. É importante destacar que cada fonte apresentava os seus totais conforme suas variáveis. Por exemplo, enquanto o OpenDOAR listou 92 repositórios, o ROAR apresentou o total de 158 repositórios brasileiros, mas incluiu revistas científicas, agregadores e plataformas de publicação de trabalhos em eventos os quais não foram considerados na contagem nesta pesquisa.

Dos 130 repositórios identificados foram excluídos 29 itens do cômputo geral de repositórios devido aos seguintes casos: links quebrados, repositórios cadastrados por setores diferentes da mesma instituição ocasionando a duplicação, erro de categorização (dois sites listados erroneamente como Repositório e um repositório da Colômbia atribuído ao Brasil) e ainda repositórios exclusivos de objetos de aprendizagem e banco de dados que nesse estudo não foram caracterizados como publicação científica. 0 quadro 1 apresenta os casos excluídos da contagem geral. Assim, dos 130 repositórios levantados nas fontes, foram analisados 101 que estavam ativos representando quase $78 \%$ do total identificado.

Quadro 1: Repositórios excluídos do levantamento.

\begin{tabular}{|l|c|}
\multicolumn{1}{|c|}{ Repositórios excluídos } & Ocorrências \\
\hline Link Quebrado & 19 \\
\hline Duplicados & 3 \\
Categorizado de forma errada & 3 \\
Repositórios de Objetos de aprendizagem & 3 \\
\hline Repositório de Banco de dados & 1 \\
\hline TOTAL & 29 \\
\hline
\end{tabular}

Fonte: Dados da pesquisa (2017).

Devido às suas diferentes características observadas ao longo do processo de coleta de dados, foi preciso categorizar e definir os repositórios de acordo com alguns parâmetros observados no campo com a finalidade de contemplar os diferentes casos e facilitar a análise conforme os termos abaixo.

É preciso esclarecer, no entanto, que está categorização não tem por objetivo alterar as nomenclaturas conhecidas na literatura em relação aos repositórios. Trata-se apenas de demonstrar os problemas em relação ao conteúdo especialmente em relação ao baixo número de artigos ou à ausência de artigos nos repositórios e à incapacidade de se fazer buscas por tipo de item, incluindo artigos, em função de problemas relativos ao planejamento e gestão do repositório verificados durante as análises.

a) Repositório Temático - é um repositório especializado em uma área do conhecimento que possui artigos de periódicos entre outros tipos de materiais aproximandose da abordagem flexível nos termos definidos por Leite (2009);

b) Repositório temático com artigos mesclados - é um repositório especializado em uma área do conhecimento que possui artigos de periódicos entre outros tipos de materiais, mas não é possível contá-los, pois a coleção de artigos de periódicos está mesclada com outra coleção;

c) Repositório temático sem artigos - é um repositório especializado em uma área do conhecimento que não possui artigos de periódicos, mas contém outros tipos de documentos. Isto é, não foi planejado a princípio para armazenar artigos de periódicos.

d) Repositório Institucional - é um repositório que contem a produção gerada pela comunidade que faz parte da instituição incluindo vários tipos de documentos desde artigos, capítulos de livros, trabalhos apresentados em eventos até teses e dissertações, entre outros tipos de publicações aproximando-se da abordagem flexível nos termos definidos por Leite (2009).

e) Repositório institucional com poucos ou nenhum artigo - é um repositório que contém diversos tipos de produção gerada pela comunidade que faz parte da instituição, mas o número de artigos é da ordem de 0 a 5 . Ou seja, a coleção de artigos de periódicos está zerada ou possui poucos documentos. Porém, originalmente o repositório foi planejado para incluir artigos de periódicos. 
f) Repositório institucional sem artigo - é um repositório que contém a produção gerada pela comunidade que faz parte da instituição, mas não contem qualquer artigo de periódicos, mas contém outros tipos de documentos.

g) Repositório institucional de Teses \& Dissertações - é um repositório institucional que reúne teses e dissertações defendidas na instituição de origem e é dedicado somente a esse tipo de coleção, isto é, não possui artigos de periódicos.

h) Repositório institucional com artigos mesclados - é um repositório que contém diversos tipos de produção gerada pela comunidade que faz parte da instituição incluindo artigos de periódicos. Porém, não é possível contá-los pois a coleção de artigos está mesclada com outra coleção.

i) Repositório institucional híbrido - é um repositório que contém diversos tipos de produção gerada pela comunidade que faz parte da instituição. Porém, uma parte das coleções exige login e senha para acesso ao texto integral.

j) Repositórios institucionais de acesso restrito - é um repositório que contém diversos tipos de produção gerada pela comunidade que faz parte da instituição, mas, exige login e senha para acesso ao texto integral.

k) Biblioteca Digital - é uma biblioteca digital que contém a produção institucional e/ou coleções bibliográficas digitalizadas.

\subsection{Número de artigos e volume total de itens:}

Em relação à primeira variável foi possível verificar que do total da produção depositada em 101 repositórios, 55 repositórios categorizados como Repositório Institucional (item d) concentraram a maior parte da produção representando $54.50 \%$ do total conforme demonstrado no quadro 2 e 3. Considerando os dados levantados, é possível afirmar que o Acesso Aberto Verde nos termos da BOAI é representado por essa categoria que concentra $97.50 \%$ do total de artigos presentes nos 101 repositórios identificados. 0 estudo de Costa e Leite (2017) também observou a concentração maior de repositórios com artigos na Região da América Latina e Caribe embora com universo de estudo e respectivo percentual menor. Também é preciso destacar que cerca de $70 \%$ dos repositórios do mundo cadastrados no OpenDOAR contém artigos em suas coleções reforçando a vocação dos repositórios no cumprimento do Acesso Aberto Verde. Dessa forma, o que foi preconizado pelo Relatório Finch (FINCH et al., 2012) isto é, que caberiam aos repositórios apenas preservar literatura cinzenta - não se configura no Brasil, nem na Região da América Latina e Caribe segundo estudos de Costa e Leite (2017) nem tão pouco no mundo, segundo dados do OpenDOAR.

Dentre os 46 repositórios restantes (45,5\%) que não tem artigos ou possui poucos artigos, $11,9 \%$ do total de repositórios dedicam-se exclusivamente às teses e dissertações e $10,9 \%$ são repositórios que previram coleções de artigos de periódicos, mas ainda não possuem esses itens (ou são poucos). Os outros 3 repositórios $(2,9 \%)$ possuem restrições de acesso.

Dessa forma, restaram 9,8\% do total de repositórios que colecionam outros tipos de coleções que não artigos. Ainda assim, seria importante verificar quais desses repositórios possuem potencial para contribuir para o fortalecimento do Acesso Aberto Verde no país e quais realmente não apresentam essa vocação. Estratégias e diretrizes nacionais poderiam levar isso em consideração para aumentar o número de repositórios em pleno funcionamento.

Quadro 2: Dados totais dos Repositórios com as variáveis.

\begin{tabular}{|l|l|l|l|l|}
\multicolumn{1}{|c|}{ Variáveis } & $\begin{array}{l}\text { Total de } \\
\text { repositórios }\end{array}$ & $\begin{array}{c}\text { \% do total de } \\
\text { repositórios }\end{array}$ & $\begin{array}{l}\text { Total de artigos } \\
\text { nos repositórios }\end{array}$ & $\begin{array}{c}\text { Total de itens } \\
\text { do repositório }\end{array}$ \\
\hline a) Repositório Temático & 1 & 1,0 & 442 & 8.148 \\
\hline $\begin{array}{l}\text { b) Repositório Temático } \\
\text { com artigos mesclados } \\
\text { c) Repositório Temático } \\
\text { sem artigos }\end{array}$ & 1 & 1,0 & $\begin{array}{l}\text { Não há como } \\
\text { quantificar } \\
0\end{array}$ & 317 \\
\hline d) Repositório Institucional & 55 & 1,9 & 2.769 \\
\hline
\end{tabular}




\begin{tabular}{|c|c|c|c|c|}
\hline Variáveis & \begin{tabular}{|l} 
Total de \\
repositórios
\end{tabular} & $\begin{array}{l}\text { \% do total de } \\
\text { repositórios }\end{array}$ & $\begin{array}{l}\text { Total de artigos } \\
\text { nos repositórios }\end{array}$ & $\begin{array}{l}\text { Total de itens } \\
\text { do repositório }\end{array}$ \\
\hline $\begin{array}{l}\text { e) Repositório institucional } \\
\text { com poucos ou nenhum } \\
\text { artigo }\end{array}$ & 11 & 10,9 & 6 & 53.184 \\
\hline $\begin{array}{l}\text { f) Repositório institucional } \\
\text { sem artigo }\end{array}$ & 8 & 7,9 & 0 & 279.764 \\
\hline $\begin{array}{l}\text { g) Repositório institucional } \\
\text { de Teses \& Dissertações }\end{array}$ & 12 & 11,9 & 0 & 111.747 \\
\hline $\begin{array}{l}\text { h) Repositório institucional } \\
\text { com artigos mesclados }\end{array}$ & 4 & 4,0 & $\begin{array}{l}\text { Não há como } \\
\text { quantificar }\end{array}$ & 142.025 \\
\hline $\begin{array}{l}\text { i) Repositório institucional } \\
\text { híbrido }\end{array}$ & 1 & 1,0 & 9578 & 51.511 \\
\hline $\begin{array}{l}\text { j) Repositórios } \\
\text { institucionais de acesso } \\
\text { restrito }\end{array}$ & 2 & 1,9 & $\begin{array}{l}\text { Não há como } \\
\text { quantificar }\end{array}$ & 100.354 \\
\hline k) Biblioteca Digital & 4 & 4,0 & 0 & 277.384 \\
\hline TOTAL & 101 & 100,00 & 412.373 & 2.081 .065 \\
\hline
\end{tabular}

Fonte: Dados da pesquisa (2017).

No quadro 2 também é possível verificar a baixa proporção entre os valores totais dos artigos de periódicos em relação ao total de itens presentes nos repositórios indicando uma forte tendência da abordagem flexível nos repositórios brasileiros.

0 quadro 3, apresenta a condensação das onze categorias em três conforme descrito acima nas alíneas 'a' até ' $\mathrm{k}$ ' do quadro 2 com a finalidade de simplificar a visualização do cenário.

Quadro 3: Distribuição resumida dos totais de todos os repositórios.

\begin{tabular}{|l|l|l|l|l|}
\hline \multicolumn{1}{|c|}{ Variáveis } & $\begin{array}{c}\text { Total de } \\
\text { repositórios }\end{array}$ & $\begin{array}{c}\text { \% do total de } \\
\text { repositórios }\end{array}$ & $\begin{array}{c}\text { Total de artigos } \\
\text { nos repositórios }\end{array}$ & $\begin{array}{c}\text { Total de itens } \\
\text { do repositório }\end{array}$ \\
\hline $\begin{array}{l}\text { Repositórios Institucionais } \\
\text { (d) }\end{array}$ & 55 & 54,5 & 402.347 & 1.053 .862 \\
\hline $\begin{array}{l}\text { Repositórios sem artigos } \\
\text { ou com poucos artigos (a-c, } \\
\text { e-h, k) }\end{array}$ & 43 & 42,6 & 448 & 875.338 \\
\hline $\begin{array}{l}\text { Repositórios que } \\
\text { apresenta algum tipo de } \\
\text { restrição de acesso (i-j) }\end{array}$ & 3 & 2,9 & $>9578^{+}$ & 151.865 \\
\hline TOTAL & 101 & 100,0 & 412.373 & 2.081 .065 \\
\hline
\end{tabular}

Fonte: Dados da pesquisa (2017).

Legenda: + Não foi possível quantificar o número de artigos de dois repositórios pois os artigos estavam mesclados nas coleções com outros tipos de produção científica.

Outra característica que pode ser observada na compilação dos dados refere-se à distribuição dos repositórios por região do país. Destaque para a liderança do Sudeste seguida pela região Sul e Centro-Oeste conforme destacado no quadro 4.

Quadro 4: Distribuição dos Repositórios por região do país

\begin{tabular}{|l|c|c|} 
Regiões do país & Total de repositórios & $\mathbf{\%}$ \\
Sudeste & 44 & 43 \\
Sul & 20 & 20 \\
\hline Centro-Oeste & 19 & 19 \\
\hline Nordeste & 15 & 15 \\
Norte & 3 & 3 \\
Total & 101 & 100 \\
\hline
\end{tabular}

Fonte: Dados da pesquisa (2017). 


\subsection{Tipo de financiamento}

A segunda variável verifica tipos de financiamento dos repositórios e foi observado que do número total de repositórios levantados, 33 receberam recursos provenientes do Edital da FINEP os quais foram categorizados conforme segue no quadro 5.

Esses repositórios representam $20 \%$ do total de artigos de periódicos depositados nos 101 repositórios identificados e um pouco mais de $20 \%$ do total da produção de repositórios. Chama a atenção a baixa representatividade dos repositórios em relação ao número de itens depositados apesar dos recursos recebidos pelo Edital da Finep. Praticamente o total de artigos (99.40\%) estão concentrados em $63.60 \%$ dos 33 repositórios os quais pertencem à abordagem do Acesso Aberto Verde - isto é, possuem artigos em suas coleções.

Não foi possível verificar a existência de outras fontes de financiamento nos demais repositórios pois faltam dados nos repositórios que descrevam informações como essas. Por isso, os dados de financiamento deste estudo se restringiram aos coletados a partir do mesmo documento que serviu de fonte de identificação de repositórios brasileiros (lista das universidades com o resultado do Edital da Finep conforme 3.2b) constituindo-se em uma importante limitação do estudo. A exceção é para um repositório temático da área de música que recebeu recursos pela Lei de Incentivo Fiscal. Porém, o repositório não contém artigos científicos (variável c do quadro 2). Por outro lado, a partir do caso brasileiro, é possível inferir que o investimento realizado nos repositórios é provido essencialmente pelas próprias instituições.

\begin{tabular}{|c|c|c|c|}
\hline Variáveis & $\begin{array}{l}\text { Total de } \\
\text { repsitórios }\end{array}$ & Total de artigos & $\begin{array}{l}\text { Total de itens do } \\
\text { repositório }\end{array}$ \\
\hline d) Repositório Institucional & 20 & 82.122 & 319.245 \\
\hline $\begin{array}{l}\text { e) Repositório institucional com poucos } \\
\text { ou nenhum artigo }\end{array}$ & 5 & 2 & 17.993 \\
\hline f) Repositório institucional sem artigo & 2 & 0 & 9.655 \\
\hline $\begin{array}{l}\text { g) Repositório institucional de Teses \& } \\
\text { Dissertações }\end{array}$ & 1 & 0 & 315 \\
\hline $\begin{array}{l}\text { h) Repositório institucional com artigos } \\
\text { mesclados }\end{array}$ & 1 & 464 & 841 \\
\hline Links quebrados & 4 & - & - \\
\hline TOTAL & 33 & 82.588 & 434.940 \\
\hline
\end{tabular}

Quadro 5: Repositórios financiados pelo Edital da FINEP

Fonte: Dados da pesquisa (2017).

Dentre os 101 repositórios, um terço (34) seguramente recebeu subsídios diretos ou indiretos do governo brasileiro - seja por renúncia fiscal seja por meio editais governamentais com os dados dos 33 repositórios contemplados pelos editais da Finep.

\subsection{Indicador de qualidade}

Dentre os 101 repositórios institucionais identificados 55 foram qualificados pelo The Ranking Web of World Repositories em sua edição de janeiro de 2017 representando um pouco mais de $54 \%$ do total conforme demonstrado no quadro 6 .

É importante destacar que 35 repositórios deste total são aderentes à abordagem do Acesso Aberto Verde nos termos definidos pelo BOAI (item d do quadro 3) representando cerca de 34,6\% em relação ao total de 101 repositórios.

Os 46 restantes, cerca de $45,55 \%$ do total, não aparecem no ranqueamento o que pode denotar problemas de visibilidade e impacto - os principais critérios considerados pela fonte The Ranking Web of World Repositories. Esse indicador bastante expressivo é um alerta para os problemas de gestão interna dos repositórios que precisam melhorar seus procedimentos para garantir maior visibilidade e impacto - a principal função de um repositório segundo a BOAI. 
Quadro 6: Repositórios contemplados no The Ranking Web of World Repositories

\begin{tabular}{|l|c|c|}
\multicolumn{1}{|c|}{ Variáveis } & $\begin{array}{c}\text { Total de } \\
\text { repositórios }\end{array}$ & \% \\
\hline d) Repositório Institucional & 35 & 34,6 \\
\hline g) Repositório institucional de Teses \& Dissertações & 9 & 8,9 \\
\hline f) Repositório institucional sem artigo & 4 & 3,9 \\
h) Repositório institucional com artigos mesclados & 3 & 2,9 \\
\hline e) Repositório institucional com poucos ou nenhum artigo & 2 & 1,9 \\
\hline c) Repositório Temático sem artigos & 1 & 0,9 \\
k) Biblioteca Digital & 1 & 0,9 \\
a) Repositório Temático, b) Repositório Temático mesclado, i) & 0 & 0 \\
Repositório institucional híbrido & & 55 \\
Total dos repositórios contemplados pelo ranking & 101 & 100,0 \\
Total de repositórios do estudo & & 54,4 \\
\hline
\end{tabular}

Fonte: Dados da pesquisa (2017).

Durante a coleta de dados foi possível observar a falta de uniformidade nas informações sobre os repositórios. Na maioria dos casos, a partir da observação direta não há como gerar indicadores para o país ou região da América Latina e Caribe de dados sobre data de criação do repositório, informações sobre o tipo de financiamento recebido ao longo de sua existência, número total de itens, número total de itens por tipo de documento, número total de itens por grandes áreas do conhecimento, política mandatória, política de submissão e funcionamento, dentre outros.

A maioria desses dados só poderá ser obtido de forma parcial nos diretórios citados no estudo. Para se obter um panorama completo deste cenário sempre dinâmico somente por meio de um survey a ser empreendido em escala nacional.

As discrepâncias são evidentes ao acessar os diretórios que indicam o total de artigos nos repositórios brasileiros. O OpenDOAR por exemplo lista um pouco mais de 2.300 artigos (dados de maio de 2017) quando o estudo levantou o total de 412.373 artigos presentes em repositórios brasileiros.

São dados de alta relevância que podem agregar valor ao Acesso Aberto Verde e fortalecer o Movimento de Acesso Aberto no país, na Região da América Latina e Caribe e no mundo.

\section{CONSIDERAÇÕES FINAIS}

Além de descrever as origens e situação atual do acesso aberto no Brasil e, em particular, do Acesso Aberto Verde, o presente estudo procurou identificar os repositórios existentes em diversas fontes de forma exaustiva, por meio de um levantamento que pudesse retratar a situação até maio de 2017. Foi comprovado que os diretórios que listam repositórios do mundo todo não expressa a realidade que apresenta um número bastante considerável para mais. Os 101 repositórios identificados estão em pleno funcionamento e apresentam características distintas conforme foi verificado por meio da observação direta.

Foi observado que 54,5\% dos 101 repositórios identificados estão alinhados ao Acesso Aberto Verde concentrando 97,5\% do total de artigos presentes em repositórios brasileiros. Guardadas as devidas proporções, esses resultados acompanham a tendência da Região da América Latina e Caribe e também no mundo em relação ao número expressivo de repositórios que contem artigos científicos comparados a outros tipos de repositórios que não possuem artigos. Os números podem melhorar a partir da busca pela qualidade dos repositórios aumentando sua visibilidade e impacto incluindo a necessidade de diretrizes nacionais para boas práticas. Esta necessidade ficou evidente ao ser observado que a abordagem flexível se destacou como principal característica dos repositórios brasileiros. De acordo com Leite (2009) a perspectiva flexível vai exigir maior complexidade no processo de gestão do repositório seja em termos de espaço para armazenamento, padrões de metadados para diferentes coleções e formatos entre outros aspectos indicando, portanto, a importância de elaboração de diretrizes em nível nacional para o processo de gestão de repositórios no país. 
Outra característica observada refere-se à concentração de repositórios na região sudeste (43\% do total), seguida pela região Sul (20\%) e Centro-Oeste (19\%).

No Brasil apenas 9,8\% dos repositórios não possuem artigos embora contenham vários outros tipos de coleções incluindo teses e dissertações, trabalhos apresentados em eventos, etc.

É importante observar que foram excluídos desse percentual os repositórios dedicados à teses e dissertações em função de seu protagonismo entre as publicações científicas (apesar de não ser artigo). Foi possível observar a necessidade de uma política nacional ou diretriz para orientar as universidades na organização desse tipo de produção. Neste estudo, os repositórios de teses e dissertações representam 11,9\% do total.

Também não foram considerados daquele percentual de 9,8\% os repositórios que foram planejados para receber artigos de periódicos, mas tem pouco ou nenhum artigo depositado. Os repositórios desta categoria representam 10,9\% do total e ainda não cumprem a missão da abordagem do Acesso Aberto Verde com baixo depósitos de artigos.

Por outro lado, cerca de $45,5 \%$ dos repositórios brasileiros não aparecem no The Ranking Web of World Repositories denotando problemas internos para alavancar a visibilidade, impacto e reconhecimento desses repositórios no cenário nacional e mundial. É um número bastante expressivo indicando a necessidade de um esforço dos gestores para superar as dificuldades institucionais. Um repositório não cumpre sua missão se não alcança visibilidade e impacto.

A impossibilidade de se fazer buscas por todos os tipos de publicações existentes nos repositórios foi outro agravante identificado devido às coleções "mescladas" conforme descrito nas variáveis "b" e " $h$ " referentes aos repositórios temáticos e institucionais com artigos "mesclados" com outros tipos de coleções. Considerando que o artigo científico é o principal foco do Acesso Aberto Verde, repositórios planejados desta forma não contribuem para o avanço do Movimento do Acesso Aberto.

Outro aspecto negativo observado refere-se à falta de informação nos sites dos repositórios sobre financiamento ou captação de recursos. É possível inferir que as instituições de origem oferecem os recursos necessários para a implantação e manutenção dos repositórios uma vez que estes continuam no ar e vem sendo atualizados. Por outro lado, considerando que o sucesso de um repositório é dependente do apoio institucional - seja em termos de recursos humanos e materiais seja em termos do envolvimento político institucional requerido - essa variável pode afetar de forma negativa a manutenção dos repositórios em longo prazo, sendo portanto, um aspecto bastante delicado para os gestores de repositórios brasileiros, em particular e para o Acesso Aberto Verde em geral. Neste estudo foi verificado que um terço dos repositórios receberam subsídios governamentais (direto ou indireto) mas aparentemente não se distinguiram dos demais que não receberam esses recursos já que representam $20 \%$ da produção de artigos. É preciso destacar que uma das fontes de identificação de repositórios brasileiros foi exatamente o edital da Finep e certamente este fato se caracteriza em uma limitação do estudo.

Apesar da introdução do Acesso Aberto no Brasil ter gerado bons frutos com indicadores que destacam sua liderança no Movimento do Acesso Aberto - oitavo lugar em número de repositórios - e na sua participação crescente em pesquisa e desenvolvimento, foram observados algumas dificuldades que exigem atenção tanto da parte dos gestores de repositórios brasileiros quanto das instituições e autoridades governamentais que lideram o acesso aberto no país.

Também é preciso considerar que ainda não foi superada a estrutura que separa a Ciência Mainstrain da Ciência Periférica (GUÉDON, 2010) exigindo do governo brasileiro um planejamento nacional envolvendo todas as instituições que produzem conhecimento científico e/ou acadêmico no país para pensar em estratégias próprias com o objetivo de vencer tais obstáculos.

Dessa forma, ações coordenadas poderiam ser empreendidas para orientar as diversas propostas de forma integrada e permanente alcançando todos os participantes do processo em nível nacional. Um comitê gestor supra-institucional poderia ser responsável por estabelecer e empreender essas ações de forma a facilitar a coordenação e implementação das mesmas. 0 comitê ou comissão também poderá contribuir para:

a) estabelecer as boas práticas na implementação e manutenção de repositórios de forma que sejam contempladas principalmente: cadastro do repositório em diretórios de 
acesso aberto, disponibilidade de dados sobre a data de criação do repositório, sobre o tipo de financiamento recebido ao longo de sua existência, número total de itens, número total de itens por tipo de documento, número total de itens por grandes áreas do conhecimento, de forma sistemática e atualizada, política mandatória, política de submissão e funcionamento, em quais situações são aplicáveis a criação de repositórios institucionais dedicados a um tipo de documento, etc.

b) contribuir para o planejamento de pesquisas do tipo survey em nível nacional para coletar dados que possam retratar a situação atual dos repositórios de forma exaustiva com o objetivo de gerar indicadores para um planejamento coordenado ou para a elaboração de política nacional para os repositórios brasileiros.

c) levar para os fóruns existentes sobre repositórios a necessidade de contemplar o fomento de diretrizes nacionais para alinhar os repositórios ao Acesso Aberto Verde preconizado pela BOAI e para, sobretudo, conscientizar as pessoas sobre sua importância e papel no processo de comunicação científica.

Vários estudos latino-americanos em especial Babini e Machin-Mastromatteo (2015) e Costa e Leite (2017) são unânimes nessa reivindicação de ações regionais e integradas para formalização de políticas e programas nacionais de acesso aberto, fomento do debate público sobre o tema, promoção de boas práticas, treinamento e investimento em infraestrutura. Certamente que a missão é bastante complexa por envolver múltiplos participantes, instituições de diversas naturezas bem como diferentes países. Por outro lado, este estudo demonstra que os passos dados até aqui, alteraram de alguma forma o cenário de isolamento do país na Ciência Mainstrain.

Porém, novos passos precisam ser dados. No que se refere aos repositórios é importante observar que a etapa inicial de implantação de repositórios liderada pelo governo brasileiro - incluindo as instituições de ensino superior e de pesquisa - já foi concluído. Agora, novas ações precisam ser formuladas com a mobilização forte dos envolvidos para que seja possível desencadear um novo estágio de desenvolvimento dos repositórios - avançar e fortalecer o Acesso Aberto Verde no país, na Região da América Latina e Caribe e no mundo.

\section{FINANCIAMENTO}

A pesquisa que recebeu apoio da Universidade Federal do Estado do Rio de Janeiro (UNIRIO) por meio do Programa Pesquisador UNIRIO Produtividade em Pesquisa Bolsa de Produtividade-PROPG (PQ-UNIRIO) -AUXÍLIO PESQUISA no 001/2016.

\section{AGRADECIMENTO}

Nossos sinceros agradecimentos: às estudantes de graduação em Biblioteconomia da UNIRIO pelo apoio na coleta de dados desta pesquisa: Poliana Ribeiro Teixeira, Julianne da Silva Ferraz e Luane Neves de Souza Porto - a primeira como bolsista de Iniciação Científica e as demais como voluntárias; e ao Professor Doutor Alexandre Sousa da Silva do Grupo de Apoio Estatístico (GAE) da UNIRIO. Na oportunidade agradecemos também aos avaliadores.

\section{REFERÊNCIAS}

ALBAGLI, Sarita. Ciência aberta em questão. In: ALBAGLI, Sarita; MACIEL, Maria Lucia; ABDO, Alexandre Hannud (Orgs.). Ciência aberta, questões abertas. Brasília: IBICT; Rio de Janeiro: Unirio, 2015. p. 9-25. Disponível em: <http://livroaberto.ibict.br/bitstream/1/1060/1/Ciencia\%20aberta_questoes\%20abertas_ PORTUGUES_DIGITAL\%20(5).pdf>. Acesso em: 24 out. 2018.

ALPERIN, J. P.; FISCHMAN, G.; WILLINSKY, J. Open access and scholarly publishing in Latin America: ten flavours and a few reflections. Liinc em revista, Rio de Janeiro, v. 4, n/2, 2008. 
BABINI, Dominique; MACHIN-MASTROMATTO, Juan D. Latin American science is meant to be open access Initiatives and current challenges. Information Development, v, 31, n. 5, p. 477- 481, 2015.

BUDAPEST OPEN ACCESS INITIATIVE. Read the Budapest Open Access Initiative. 2002. Disponível em: <http://www.budapestopenaccessinitiative.org/read>. Acesso em: 25 maio. 2017.

BLOG DOS REPOSITÓRIOS BRASILEIROS. Repositórios brasileiros de acesso aberto serão lançados amanhã. 2010. Disponível em: <https://blogrib.wordpress.com/2010/10/14/repositorios-brasileiros-de-acesso-abertoserao-lancados-amanha/>. Acesso em 25 maio 2017.

BRUMATTI, J. D. 0 acesso aberto verde no Brasil: um estudo descritivo da produção científica depositada em repositório institucional. 2016. 119 f. Dissertação (Mestrado Profissional em Biblioteconomia)-Universidade Federal do Estado do Rio de Janeiro, Rio de Janeiro, 2016. Disponível em: <http://www.unirio.br/ppgb/arquivo/josimara-diasbrumati>. Acesso em 11 mar. 2017.

CALHOUN, Karen. Exploring digital libraries: foundation, practices, prospects. London: Facet Publishing, 2014.

CARVALHO, Ana Maria Ferreira de. Análise do uso de conteúdo em repositórios institucionais de instituições de ensino superior ibero-americanas: um estudo cientométrico na base Scopus. 2018. Tese (Doutorado em Ciência da Informação)Universidade Federal do Rio de Janeiro, Rio de Janeiro, 2018.

CONSEJO SUPERIOR DE INVESTIGACIONES CIENTÍFICAS (Espanha). Ranking web of world repositories. Madrid, 2012. Disponível em:

<http://repositories.webometrics.info/en/world>. Acesso em: 25 maio. 2017.

COSTA, Michelli Pereira da; LEITE, Fernando César Lima. Repositórios institucionais da América Latina e o acesso aberto. Brasília: IBICT, 2017. 178 p. Disponível em: <http://eprints.rclis.org/31109/1/Repositorio\%20America\%20Latina_Digital.pdf>. Acesso em 25 maio 2017.

COSTA, S. M. S.; KURAMOTO, H.; LEITE, Fernando César Lima. Acesso aberto no Brasil: aspetos históricos, ações institucionais e panorama atual. In: RODRIGUES, E.; SWAN, A.; BAPTISTA, A. A. (Org.). Uma década de acesso aberto na UMinho e no mundo. Braga: Universidade do Minho, Serviços de Documentação, 2013. p. 133-150. Disponível em: <http://repositorium.sdum.uminho.pt/bitstream/1822/26144/3/RepositoriUM_10anos.pdf >. Acesso em: 25 maio. 2015.

DSPACE FOUNDATION. Dspace user registry. [Ithaca, 2010]. Disponível em: <http://registry.duraspace.org/registry/dspace>. Acesso em: 25 maio. 2017.

ELSEVIER. World of Research 2015. Amsterdam, 2015. Disponível em: <http://cdn.elsevier.com/promis_misc/ELS-WOR2015.pdf>. Acesso em: 25 maio. 2017.

FINCH, Dame Janet et al. Accessibility, sustainability, excellence: how to expand access to research publications, 2012. Disponível em: <https://www.acu.ac.uk/research-informationnetwork/finch-report-final>. Acesso em: 24 out. 2018.

GARGOURI, Yassine; LARIVIÈRE, Vincent; GINGRAS, Yves; CARR, Les; HARNAD, Stevan. Green and Gold Open Access Percentages and Growth, by Discipline. [2012?]. Disponível em: <https://arxiv.org/abs/1206.3664>. Acesso em: 10 ago. 2018. 
GIGLIA, E.; HARNAD, S. Open Access 2012: achievements,further steps, and obstacles: an interview with Stevan Harnad. European Journal of Physical and Rehabilitation Medicine, v. 48, n. 3, 2012. Disponível em:

<http://www.minervamedica.it/en/getfreepdf/IQzEb492wud2Sz6cPa92c3mgbM67BYFE44 xGs5Jq28n3NmHNijPVAK5CMJb5kJ9QVAnUsnFqQ2wZd15IiPQbBA\%253D\%253D/R33Y201 2N03A0535.pdf>. Acesso em: 25 maio. 2017.

GUÉDON, Jean-Claude. The "Green" and "Gold" Roads to Open Access: The Case for Mixing and Matching. Serials Review, 2004. Disponível em:

<https://depts.washington.edu/uwbri/PDF\%20Files/The\%20green\%20and\%20gold\%20ro ads $\% 20$ to $\% 20$ open $\% 20$ access-the $\% 20$ case $\% 20$ for $\% 20$ mixing $\% 20$ and $\% 20$ matching.pdf $>$. Acesso em: 25 maio 2017.

GUÉDON, Jean-Claude. Acesso aberto e divisão entre ciência predominante e ciência periférica.In: FERREIRA, S. M. S. P.;TARGINO, M. G. Acessibilidade e visibilidade de revistas científicas eletrônicas. São Paulo: SENAC, 2010. p. 21-77.

HARNAD, Stevan. Why the UK Should Not Heed the Finch Report. LSE Impact Blog. 2012. Disponível em: <http://blogs.lse.ac.uk/impactofsocialsciences/2012/07/04/why-the-ukshould-not-heed-the-finch-report/>. Acesso em: 10 ago. 2018.

HARNAD, Stevan; CARR, Les; GINGRAS, Yves. Maximizing research progress through Open Access mandates and metrics. Liinc em revista, Rio de Janeiro, v. 4, n. 2, 2008. Disponível em: <http://revista.ibict.br/liinc/article/view/3165/2831>. Acesso em: 25 maio 2017.

HARNAD, Stevan et al. The Access/Impact Problem and the Green and Gold Roads to Open Access. Serials Review,v. 30, n. 4, p. 310-314, 2004. Disponível em:

<https://eprints.soton.ac.uk/260209/1/impact.html>. Acesso em: 25 maio 2017.

INSTITUTO BRASILEIRO DE INFORMAÇÃO EM CIÊNCIA E TECNOLOGIA. 8a Conferência Luso-Brasileira sobre Acesso Aberto (ConfOA). Brasília, 2017. Disponível em: $<$ http://www.ibict.br/Sala-de-Imprensa/eventos/8a-conferencia-luso-brasileira-sobreacesso-aberto-confoa/impressao>. Acesso em: 23 abr. 2017.

INSTITUTO BRASILEIRO DE INFORMAÇÃO EM CIÊNCIA E TECNOLOGIA.Ibict lança Manifesto de Acesso Aberto a Dados da Pesquisa Brasileira para Ciência Cidadã. Rio de Janeiro, 2016. Disponível em: <http://www.ibict.br/Sala-de-Imprensa/noticias/2016/ibictlanca-manifesto-de-acesso-aberto-a-dados-da-pesquisa-brasileira-para-ciencia-cidada > . Acesso em: 2 abr. 2017.

INSTITUTO BRASILEIRO DE INFORMAÇÃO EM CIÊNCIA E TECNOLOGIA. Ibict lança manifesto pelo acesso livre à informação científica. Brasília, 2005. Disponivel em: $<$ http://www.ibict.br/Sala-de-Imprensa/noticias/2005/ibict-lanca-manifesto-pelo-acessolivre-a/>. Acesso em: 2 abr. 2017.

INSTITUTO BRASILEIRO DE INFORMAÇÃO EM CIÊNCIA E TECNOLOGIA. Lançado novo edital para estimular implantação de repositórios institucionais. 2009. Disponível em:< http://www.ibict.br/Sala-de-Imprensa/noticias/2009/lancado-novo-edital-para-estimularimplantacao-de/>. Acesso em: 25 maio 2017.

INSTITUTO BRASILEIRO DE INFORMAÇÃO EM CIÊNCIA E TECNOLOGIA. Lançada nova chamada do edital para estimular implantação de repositórios institucionais. 2010. Disponível em: <http://www.ibict.br/Sala-de-Imprensa/noticias/2010/lancada-novachamada-do-edital-para-

estimular/?searchterm=Lançada\%20nova\%20chamada\%20do\%20edital\%20para\%20esti mular\%20implantação\%20de\%20repositórios\%20institucionais>. Acesso em: 25 maio 2017. 
LA REFERENCIA. Somos La Referencia. [Santiago, 2018]. Disponível em: $<$ http://www.lareferencia.info/joomla/pt/institucional/quienes-somos >. Acesso em: 11 jun. 2018.

LEITE, Fernando César Lima. Como gerenciar e ampliar a visibilidade da informação científica brasileira: repositórios institucionais de acesso aberto. Brasília: IBICT, 2009.

MILLINGTON, Peter. OpenDOAR and ROAR. Nottingham, University of Nottingham, 2008. Disponível em: <http://www.opendoar.org/about.html>. Acesso em: 24 out. 2017. Power point.

OPEN ACCESS SCHOLARLY INFORMATION SOURCEBOOK. Open Access and Developing Countries. [2010]. Disponível em:

<http://www.openoasis.org/index.php\%3Foption\%3Dcom_content\%26view\%3Darticle\%2 6id\%3D28\%26Itemid\%3D412>. Acesso em 21 abr. 2017.

PACKER, Abel L. MENEGUINI, Rogério. O SciELO aos 15 anos: raison d'être, avanços e desafios para o futuro. In: PACKER, Abel L. (Org.). SciELO: 15 Anos de Acesso Aberto [livro eletrônico]: um estudo analítico sobre Acesso Aberto e comunicação científica. Paris: UNESCO, 2014. p. 15-28. Disponível em: <http://www.scielo.org/local/File/livro.pdfs. Acesso em: 24 out. 2017.

PORTUGAL. Ministério da Ciência, Tecnologia e Ensino Superior. Glossário. C2016. Disponível em: < http://www.ciencia-aberta.pt/glossario>. Acesso em: 24 out. 2018.

SANTILLÁN ALDANA, Julio Francisco. Modelos de negócio dos periódicos científicos de acesso aberto na América Latina e Caribe. 2014. 246 f., il. Dissertação (Mestrado em Ciência da Informação)—Universidade de Brasília, Brasília, 2014. Disponível em: <http://repositorio.unb.br/bitstream/10482/16386/1/2014_JulioFranciscoSantillanAldana. pdf >. Acesso em: 12 mar. 2017.

SCHÖPFEL, Joachim. Open Access to Scientific Information in Emerging Countries. D-Lib Magazine, v. 23, n. 3/4, Mar./Apr. 2017. Disponível em: <https://doi.org/10.1045/march2017-schopfel>. Acesso em: 12 mar. 2017.

TZOC, Elías. El Acceso Abierto en América Latina: Situación actual y expectativas. Revista Interamericana de Bibliotecología, v.35, n.1, Medellín, jan./jun. 2012. Disponível em: < http://aprendeenlinea.udea.edu.co/revistas/index.php/RIB/article/view/13340>. Acesso em: 12 mar. 2017.

Editores do artigo: Enrique Muriel-Torrado, Edgar Bisset Alvarez, Camila Barros. 